\title{
Galactic Bulge PNe: Carbon molecules in oxygen-rich environments
}

\author{
Lizette Guzmán-Ramírez ${ }^{1}$, Albert Zijlstra ${ }^{1}$, Roisin Níchuimín ${ }^{1}$, \\ Krzysztof Gesicki ${ }^{2}$, Eric Lagadec ${ }^{3}$, Tom J. Millar ${ }^{4}$ and Paul M. Woods ${ }^{5}$ \\ ${ }^{1}$ Jodrell Bank Centre for Astrophysics, School of Physics \& Astronomy, \\ The University of Manchester, Manchester, M13 9PL, UK \\ email: lizette.ramirez@postgrad.manchester.ac.uk \\ ${ }^{2}$ Centrum Astronomii UMK, ul.Gagarina 11, 87-100 Torun, Poland \\ ${ }^{3}$ European Southern Observatory, Karl-Schwarzschild-Str 2, 85748 Garching, Germany \\ ${ }^{4}$ Astrophysics Research Centre, Queen's University Belfast, Belfast BT7 1NN, UK \\ ${ }^{5}$ Dept. of Physics and Astronomy, University College London, Gower Street, London \\ WC1E6BT, UK
}

\begin{abstract}
Galactic bulge planetary nebulae show evidence of mixed chemistry with emission from both silicate dust and PAHs. This mixed chemistry is unlikely to be related to carbon dredge up, as third dredge-up is not expected to occur in the low mass bulge stars. We show that the phenomenon is widespread, and is seen in 30 nebulae out of our sample of 40 . A strong correlation is found between strength of the PAH bands and morphology, in particular, the presence of a dense torus. A chemical model is presented which shows that hydrocarbon chains can form within oxygen-rich gas through gas-phase chemical reactions. We conclude that the mixed chemistry phenomenon occurring in the galactic bulge planetary nebulae is best explained through hydrocarbon chemistry in an UV-irradiated, dense torus.
\end{abstract}

Keywords. planetary nebulae: general, circumstellar matter, Galaxy: bulge.

\section{Introduction}

Planetary Nebulae (PNe) are remnants of the extreme mass loss experienced by asymptotic giant branch (AGB) stars. During the AGB phase, a star may evolve from being oxygen-rich to being carbon-rich, a change reflected in the chemical composition of the stellar wind. The change occurs when carbon produced by He-burning is brought to the surface by processes in the stellar interior thereby increasing the $\mathrm{C} / \mathrm{O}$ ratio, until it exceeds unity and a carbon star is formed (Herwig 2005).

In the molecular ejecta, the CO molecule locks away the less abundant element, leaving the remaining free $\mathrm{O}$ or $\mathrm{C}$ to drive the chemistry and dust formation. Oxygen-rich shells are characterized by silicate dust, while carbon-rich shells show Polycyclic Aromatic Hydrocarbon (PAH) emission bands and carbonaceous dust. This dichotomy is indeed by and large observed.

The mixed-chemistry phenomenon has been seen in PNe with late Wolf-Rayet type central stars (Waters et al. 1998), where it is attributed to the presence of old oxygen-rich material in a circumstellar disk, with the PAHs forming in a more recent carbon-rich outflow.

Perea-Calderón et al. (2009) showed that the mixed-chemistry phenomenon is widespread among the PNe in the galactic bulge (GB). Their Spitzer observations show that the simultaneous presence of oxygen and carbon-rich dust features is common, and is not restricted to objects with late/cool [WC] type stars. The traditional explanation relating the mixed chemistry to a recent evolution towards carbon-star is unlikely for the bulge objects, as these old, low-mass stars are not expected to show third dredge-up, and therefore should not show enhanced $\mathrm{C} / \mathrm{O}$ ratios. 

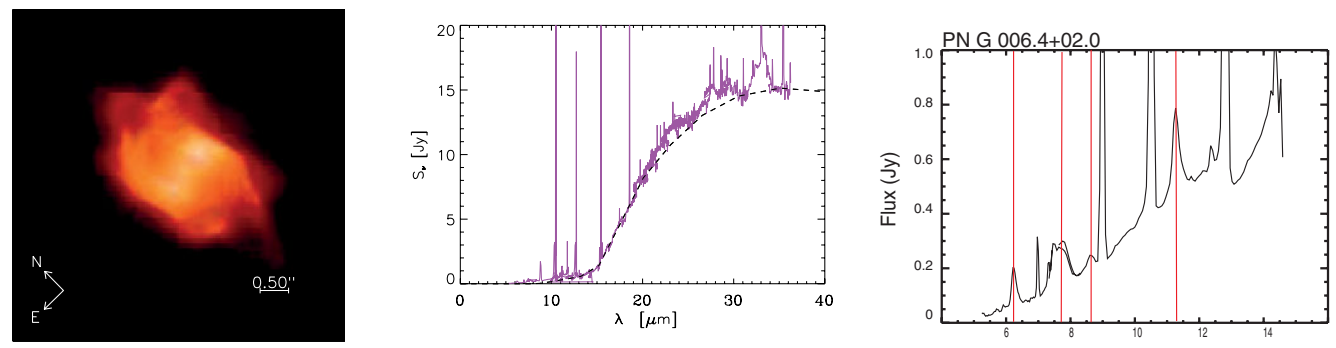

Figure 1. HST image of PN M1-31 (left),Spitzer spectrum where the dashed line is the MC3D model (centre) and the short-wavelength part of the Spitzer spectrum that shows the PAHs bands of M1-31 (right).

\section{Observations and results}

Spectra of 40 bulge PNe were taken with the Infrared Spectrograph (IRS) (Houck et al. 2004). The spectra cover a range between 5.2-37.2 $\mu \mathrm{m}$. Twenty-two of the objects were included in an HST SNAP-shot (proposal 9356, PI Zijlstra). The WFPC2 images were taken in three different filters, $\mathrm{H} \alpha, V$, and [OIII], we only used the $\mathrm{H} \alpha$ images here. VLT UVES echelle spectra were obtained for these 22 objects covering the wavelength range from 3300 to $6600 \AA$, with a resolution of 80,000 . These spectra were used to investigate the nature of the central stars, based on the presence of stellar emission lines.

The most important aspect that we found is the predominance of a very specific morphology: a dense torus with bipolar or multipolar flows. This appears to correlate well with the PAH band strength. The confining tori have a range of sizes but are always well defined. We conclude that the mixed chemistry phenomenon is strongly related to morphology, or the presence of a torus. There is no clear relation to the central star, apart from a possible amplification of the PAH bands by emission-line stars.

To test if the dust spectra can be related to the presence of a torus, we model the Spitzer spectrum for the representative PN M1-31 (PN G006.4+02.0), using the MC3D (MonteCarlo 3-D) program (Wolf et al. 1999). We used astronomical silicate as the dust component as our aim is to reproduce the shape of the continuum due to emission from these dust grains.

We used an effective temperature of the star of $57,000 \mathrm{~K}$, a luminosity of $6,500 \mathrm{~L}_{\odot}$ (Gesicki \& Zijlstra 2007), an inclination of the disk of 90 degrees (edge-on), with the distance to the galactic bulge taken as $8.5 \mathrm{kpc}$ (Schneider \& Buckley 1996).

In Fig. 1, we present the HST image for the representative PN M1-31 on the right, the Spitzer spectrum and the MC3D model in the centre and on the left image we plotted the PAHs bands on the short-wavelength part of the spectrum. In Guzman-Ramirez et al. (2011) we present the 22 HST images with their corresponding Spitzer and UVES spectra.

Our calculation shows that the dust continuum emission can be explained as arising from the dense torus using oxygen-rich dust (silicate) to explain the SED: there is no significant carbonaceous component. The fitted torus is large and massive (5000-50000 AU with a dust mass of $\left.5.5 \times 10^{-3} \mathrm{M}_{\odot}\right)$. This is a significant finding, as the traditional model for mixed chemistry poses a long-lived, compact oxygen-rich disc.

In the traditional stellar evolution model, the silicates were ejected $>10^{5} \mathrm{yr}$ ago, with the star subsequently becoming carbon-rich prior to the ejection of the main nebula (Habing 1996). In contrast, the torus of M1-31 is part of the main nebula and ejected $<10^{4}$ yr ago: at this time the ejecta were oxygen-rich. The mixed chemistry phenomenon is therefore unlikely to be related to the star becoming carbon-rich, as the chances of this occurring over such a short time scale are low, whereas the occurrence of mixed chemistry in the GB appears to be relatively common. 

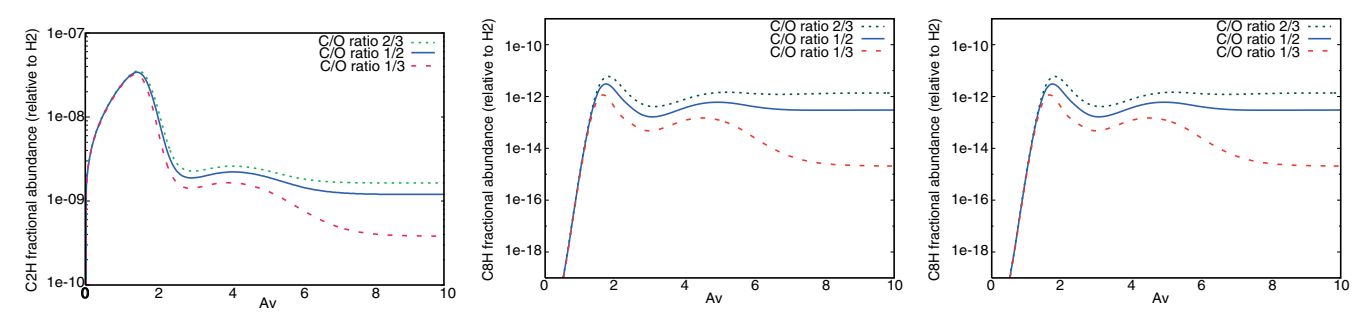

Figure 2. Calculated abundances for varying $\mathrm{C} / \mathrm{O}$ ratios for $\mathrm{C}_{2} \mathrm{H}$ (left), $\mathrm{C}_{8} \mathrm{H}$ (centre) and $\mathrm{C}_{23} \mathrm{H}_{2}$ (right).

Carbon stars originate from third dredge-up, which requires a minimum stellar mass of $M_{\mathrm{i}}>1.5 \mathrm{M}_{\odot}$ (Vassiliadis \& Wood 1993). It is not expected to occur in 10 Gyr old populations.

Furthermore, at high metallicity the formation of carbon stars is inhibited by the large oxygen abundance which needs to be overcome by the primary carbon. Carbon stars are rare in the inner Galaxy (Le Bertre et al. 2003) and almost absent in the galactic bulge (Feast 2007;Uttenthaler et al. 2008), consistent with this expectation. This strengthens the case that the mixed chemistry is occurring in oxygen-rich gas, expelled by oxygen-rich stars.

The strong correlation between mixed chemistry and morphology, specifically, the presence of a massive torus, indicates that the chemical pathway to the PAH molecules occurs within their high density regions. These tori remain molecular for some time after the ionization of the nebula has started, due to the trapping of the ionization front and due to the shielding effects of the dust column density (Woods et al. 2005). A mini PDR (photon-dominated region) is expected in these molecular regions (Phillips et al. 2010), slowly being overrun by the advancing ionization front.

We investigate whether a C-rich chemistry can be similarly driven by UV photons penetrating the dense, oxygen-rich torus. The penetration is limited by the dust extinction.

Our model, which contains gas-phase chemistry only, is based on that of Níchuimín (2009), developed for conditions in PDR regions. It uses an extrapolation of the Meudon 2006 PDR chemistry code (Le Petit et al. 2006), and assumes a semi-infinite and constantdensity slab with a density of $2 \times 10^{4} \mathrm{~cm}^{-3}$, UV radiation enhanced by a factor of 60 over the average interstellar field and treats thermal balance self-consistently.

The elemental carbon abundance is kept constant at the standard value of $1.32 \times 10^{-4}$ relative to the total hydrogen abundance (Savage \& Sembach 1996) while the elemental oxygen abundance is varied to give a range of $\mathrm{C} / \mathrm{O}$ values of $2 / 3,1 / 2$ and $1 / 3$. The goal of the models is to study the abundances of large hydrocarbons.

Figure 2 shows the calculated fractional abundances (relative to $\mathrm{H}_{2}$ ) for the molecules $\mathrm{C}_{2} \mathrm{H}, \mathrm{C}_{8} \mathrm{H}$ and $\mathrm{C}_{23} \mathrm{H}_{2}$, respectively, as function of $A_{v}$, which measures depth into the slab, and $\mathrm{C} / \mathrm{O}$ ratio.

Two hydrocarbon peaks are seen, one at $A_{V} \sim 1.5$, and one at $A_{V} \sim 4-6$. The first peak occurs in the region in which $\mathrm{C}^{+}$and $\mathrm{H}_{2}$ are abundant with the hydrocarbon chemistry initiated by the $\mathrm{C}^{+}-\mathrm{H}_{2}$ radiative association reaction. Subsequent reactions with $\mathrm{C}$ and $\mathrm{C}^{+}$form $\mathrm{C}_{2} \mathrm{H} . \mathrm{C}_{2} \mathrm{H}$ is the main seed molecule for the formation of larger hydrocarbons and reactions continue to build up to $\mathrm{C}_{8} \mathrm{H}$.

The second peak, at higher $A_{V} \sim 4-6$, occurs where atomic carbon becomes abundant and drives chemistry through proton transfer with $\mathrm{H}_{3}^{+}$to form $\mathrm{CH}^{+}$which subsequently reacts with $\mathrm{H}_{2}$ to form simple hydrides. Here the chemistry produces a surprisingly large abundance of the biggest hydrocarbons in the model.

Under the assumptions of the chemical model, it is clear that larger hydrocarbons can form within oxygen-rich environments. It is assumed here that a mechanism exists to convert these long linear hydrocarbon chains into PAH molecules. 
In order to generate sufficient reactive $\mathrm{C}$ and $\mathrm{C}^{+} \mathrm{UV}$ photons are required to dissociate $\mathrm{CO}$ contained in the dense molecular torus. Thus, the central star needs to be hot enough to generate these photons.

Our models use a radiation field that corresponds to a star of spectral type B, or temperature around $15000 \mathrm{~K}$. This suggests that the PAHs will not form until the star is hot enough to begin to ionize the nebula. By this time, the nebula will have expanded and the extinction has dropped considerably.

The need for the torus may therefore be to provide an irradiated region dense enough to allow a photon-dominated chemistry, in a nebula old enough to allow for an ionizing star. Such a requirement would explain the close relation between PAH emission in oxygen-rich $\mathrm{PNe}$, and the presence of a dense torus.

\section{Conclusions}

- We analyzed observations of 40 PNe towards the galactic bulge using the IRS instrument on board Spitzer. We found that 30 of them present a mixed chemistry phenomenon.

- A strong correlation was found between strength of the PAH bands and morphology, in particular, the presence of a massive torus.

- We modeled the Spitzer spectrum for the representative PN M1-31. The SED can be explained using oxygen-rich (silicate) dust. Because of the age of the torus of M1-31 $\left(<10^{4} \mathrm{yr}\right)$ the mixed chemistry phenomenon is unlikely to be related to a change of composition of the stellar ejecta over time. Such a change would be unexpected in the bulge population (which does not show third dredge-up), and would be very rare over such a short period in any case, whereas the occurrence of mixed chemistry appears to be relatively common.

- For the chemical analysis of the PAH formation in an oxygen-rich environment, we used the gas-phase models from Níchuimín (2009). They cover a range of $A_{v}$ and C/O ratios of $1 / 3,1 / 2$ and $2 / 3$. Although the model does not include PAH formation, it shows that large hydrocarbons can form in oxygen-rich environments and that their abundances can be relatively large in moderately shielded regions.

- The torus observed in all of the PNe that present the mixed chemistry phenomenon could provide the dense, irradiated environment needed to form the PAHs detected.

\section{References}

Feast, M. 2007, in: F. Kerschbaum, C. Charbonnel, R. F. Wing (eds.) Why Galaxies Care About AGB Stars: Their Importance as Actors and Probes, 378, 479

Gesicki, K. \& Zijlstra, A. A. 2007, in R. Napiwotzki, Burleigh, M. R. (eds.) 15th European Workshop on White Dwarfs, 372, 41

Guzman-Ramirez, L., Zijlstra, A. A., Níchuimín, R., et al. 2011, MNRAS, 414, 1667

Habing, H. J. 1996, A\&AR, 7, 97

Herwig, F. 2005, Annual Review of Astron and Astrophys, 43, 435

Houck, J. R., Roellig, T. L., van Cleve, J., et al. 2004, ApJS, 154, 18

Le Bertre T., Tanaka M., Yamamura I., Murakami H. 2003, A\&SA, 403, 943

Le Petit, F., Nehmé, C., Le Bourlot, J., \& Roueff, E. 2006, ApJS, 164, 506

Níchuimín, R. 2009, PhD thesis, Univ. Manchester

Perea-Calderón, J. V., García-Hernández, D. A., García-Lario, P., Szczerba, R., \& Bobrowsky, M. 2009, $A \& A, 495$, L5

Phillips, J. P., Cuesta, L. C., \& Ramos-Larios, G. 2010, MNRAS, 409, 881

Savage, B. D. \& Sembach, K. R. 1996, Annual Review of Astron and Astrophys, 34, 279

Schneider, S. E. \& Buckley, D. 1996, ApJ, 459, 606

Uttenthaler, S., Hron, J., Lebzelter, T., et al. 2008, A\&A, 478, 527

Vassiliadis, E. \& Wood, P. R. 1993, ApJ, 413, 641

Waters, L. B. F. M., Beintema, D. A., Zijlstra, A. A., et al. 1998, A 6 A, 331, L61

Wolf, S., Henning, T., \& Stecklum, B. 1999, A\& A, 349, 839

Woods, P. M., Nyman, L.-Å., Schöier, F. L., et al. 2005, A\&̊A, 429, 977 